ISAHP 1999, Kobe, Japan, August 12-14, 1999

\title{
ON THE USE OF INFORMATION CRITERIA IN ANALYTIC HIERARCHY
}

\author{
Indrani Basak \\ Department of Mathematics, Penn State Altoona \\ 151 CLRC \\ 3000 Ivyside Park \\ Altoona PA $16601-3760$ \\ i8b@psu.edu
}

\begin{abstract}
In this article, we discuss how the model-selection procedures such as Akaike's information criteria (AIC) can be used for selecting the most appropriate one out of several existing statistical models in the literature. Furthermore, once the appropriate model is selected, a procedure is proposed on the basis of AIC for statistical ranking of the alternatives. This ranking procedure does not suffer from the problem of intransitivity and can be based on non-normal distribution. Through the use of a small-scale simulated judgments the patterns of decision errors for the methods based on the information criteria are compared with other existing methods.
\end{abstract}

